\title{
Safety requirements-oriented interfaces environment scheme for safety-critical system
}

\author{
Y. Li, J. Guo, Y. Yang, G. Xie \& Y. Su \\ School of Information Science and Technology, \\ Southwest Jiaotong University, China
}

\begin{abstract}
With the increasing complexity of safety-critical system and component-based development approach is widely used, focusing on the problem that the system safety is affected directly by the interfaces failure of its modules, the disadvantages of current safety scheme are analyzed, and an interfaces scheme is presented to ensure safety on system level. First, according to the interactions between safetycritical system and environment, an environment interfaces failure scenario is defined, then its effects to safety-critical system are analyzed. Second, to ensure the system safety requirements, composing the module and its environment, a safety requirements-oriented interfaces environment scheme is presented, which aims to avoid the interfaces faults that could cause a system failure and provide maintenance information when system violates safety requirements. Third, an identification algorithm to generate the safety requirements-oriented interfaces environment is presented based on model checking technology. Finally, taking the urban rail transit computer based interlocking system as an example, the safety requirements-oriented interfaces environment of a signal module is analyzed with the algorithm implemented by SCADE. The result is completely consistent with the field practical experience, which shows the feasibility and effectiveness of this scheme.

Keywords: safety-critical system, safety requirements, environment interfaces failure, safety requirements-oriented interfaces environment, computer based interlocking.
\end{abstract}




\section{Introduction}

Safety-critical system (SCS) is a system whose failure could result in human death, significant property loss or environment damage [1]. Component-based development which composing smaller independently developed components into larger components assemblies is widely used in the complex SCS development. Modules is the basic notion of component and components assemblies, which affects the system safety directly. The interfaces fault of a module can easily cause a system failure. For example, one important cause of the Three Miles Island nuclear event in 1979, is an interface which gathered environment state mistakenly [2]. The identification of relation of module interfaces and system safety can benefit the SCS safety analysis, but since the concurrency, realtime, complexity and variety of interactions with environment, there are many challenges facing this task.

The traditional safety analysis methods, like FTA, ETA, FEAM, OSHA, can be used to deduce the fault modes of modules, but are incapable of analyzing the interfaces behavior. Elmqvist J and Nadjm-Tehrani S presented a safety interfaces scheme (SIS) to guarantee SCS safety on component level. SIS presents a safety condition that SCS should assure in a specific fault scenario, depending on faults mode library. This scheme has been adopted well in Swedish strategic research foundation project and the national aerospace research program NFFP [3, 4]. Elmqvist and Nadjm-Tehrani [5] improved the scheme to analyze residual risks quantitatively. Ying and $\mathrm{Xu}$ [6] extended the scheme from single and double faults mode to multiple faults mode. Since faults modes are always accumulated during system operation phase, the exhaustiveness of identification of faults modes will affect the completeness of safety interfaces, and SIS cannot be applied well to guide interface design or system integration before system put into use.

This paper presents a safety requirements-oriented interfaces environment (SRoIE) scheme to ensure SCS safety on system level. SRoIE identifies the interfaces that should be ensured for a specific safety constraints, based on system functional model and safety properties. A algorithm to generate SRoIE is presented, including a refinement algorithm for keeping the interfaces set of SRoIE minimal and critical. At last, a module of urban rail transit computer based interlocking system is taken as an example to show the feasibility of this scheme.

\section{Module and environment interfaces fault scenario}

Modules gathers environment states through input interfaces, based on desired functional requirements, provides data or control commands to environment as outputs through output interfaces. The correctness of input interfaces will directly affects the compute result and system safety of SCS.

Definition 1. A module is a tuple:

$$
\Sigma=\left(I, Q, Q_{0}, \Delta, T, O\right)
$$


where: $I$ is input interfaces, and the input domain is denoted by $V_{I} ; Q$ is finite states set; $Q_{0} \subseteq Q$ is initial states set; $\Delta$ is finite labels set; $T \subseteq Q \times \Delta \times Q$ is transition set; $O$ is output interfaces set, and the output domain is denoted by $V_{O}$.

Modules keep uninterrupted interaction with environment, and perform its functionality under transition constraints. This process is defined as a composition of module and its environment.

Definition 2. Let $\Sigma^{\prime}=\left(I^{\prime}, Q^{\prime}, Q_{0}^{\prime}, \Delta^{\prime}, T^{\prime}, O^{\prime}\right)$ be a module, $\Sigma^{\prime \prime}=\left(I^{\prime \prime}, Q^{\prime \prime}, Q_{0}^{\prime \prime}\right.$, $\left.\Delta^{\prime \prime}, T^{\prime \prime}, O^{\prime \prime}\right)$ be an environment, the composition of module and its environment is denoted:

$$
\Sigma^{C}=\Sigma^{\prime} \| \Sigma^{\prime \prime}
$$

where: $I^{C}=I^{\prime \prime}, Q^{C}=Q^{\prime} \cup Q^{\prime \prime}, Q_{0}^{C}=Q_{0}^{\prime} \times Q_{0}^{\prime \prime}, T^{C}=T^{\prime} \times T^{\prime \prime}$, $O^{C}=O^{\prime} \cup O^{\prime \prime}-O^{\prime} \cap O^{\prime \prime}, V_{I}^{\prime} \subseteq V_{O}^{\prime \prime}$.

In general, the failure caused by invalid inputs can be avoid by taking design methods, such as fault tolerance, but the consistency of interfaces and environment states is difficult to detect. The inconsistency is an environment interfaces fault scenario and could cause a system failure.

Definition 3. Let $v_{i}$ be an environment state of a module, $v_{i}^{f}$ be the input result of $v_{i}$, environment interface fault (EIF) scenario is denoted by $\Sigma\left(v_{i} / v_{i}^{f}\right)$, where $v_{i}, v_{i}^{f} \in V_{I}, v_{i} \neq v_{i}^{f}$.

The EIF represents an inconsistency of inputs and environment states. Twotuples $\left(I_{i}, v_{i}\right) \in I \times V_{I}$ indicates the input of interface $I_{i}$ is $v_{i}$, then an interaction between SCS and environment is denoted by $\mu_{i}=\bigcup_{k=1}^{n u m}\left(I_{k}, v_{k}\right)$, where num is the total interfaces numbers of module. The interfaces whose failure could cause system violates a safety constraint $\psi$ are called key interfaces, denoted by $K I^{\psi}$.

Definition 4. safety verification model of SCS is a function:

$$
f:\left(\Sigma \| \Sigma^{\prime \prime}, v_{i}, v_{o}, \psi\right) \longrightarrow(\text { true }, \text { false })
$$

where: $\Sigma$ is a module; $\Sigma^{\prime \prime}$ is the environment of $\Sigma ; v_{i} \in V_{I}$ is environment inputs; $v_{i} \rightarrow v_{o}$ and $v_{o} \in V_{o} ; \psi \in \Psi$ is a safety constraint that SCS needed to be verified.

As different environments employ different safety requirements, safety analysis must consider the concrete environment that the system placed in, Safety verification model verifies that, in $\Sigma^{\prime \prime}, \Sigma$ whether satisfy $\psi$ with $v_{i}$ and its output $v_{o}$ or not. $\forall v_{i}, f\left(\Sigma \| \Sigma^{\prime \prime}, v_{i}, v_{o}, \psi\right)=$ true means $\Sigma$ satisfies $\psi$ in $\Sigma^{\prime \prime}$, denoted by $\left(\Sigma \| \Sigma^{\prime \prime}\right) \models \psi$.

\section{SRoIE scheme}

When SCS is in $\Sigma\left(v_{i} / v_{i}^{f}\right)$, for $v_{i}, v_{i}^{f}$ may both be in input domain, SCS fails to identify these consistency, then performs its function with $v_{i}^{f}$. The safety constraint which is satisfied in normal environment may be violated in EIF scenario. 


\subsection{Safety in EIF scenario}

The environment of SCS includes gathering environment $\Sigma^{G}$ and working environment $\Sigma^{W}$, and $\Sigma^{W}$ determines the computation result of SCS is safe or not.When input interfaces get data properly, $\Sigma^{G}=\Sigma^{W}$. But if the interfaces malfunction: $\Sigma\left(v_{i} / v_{i}^{f}\right), \Sigma^{G} \neq \Sigma^{W}$. In this situation, SCS performs its functional requirements based on $v_{i}^{f}$, but $\Sigma^{W}$ verifies the safety based on $v_{i}$, then equation (3) is changed into:

$$
f:\left(\Sigma \| \Sigma^{W}, v_{i}, v_{i}^{f} \rightarrow v_{o}, \psi\right) \longrightarrow(\text { true }, \text { false })
$$

An underlying risk is posed that SCS performed its functional requirements successfully, and ensured the safety in $\Sigma^{G}$, but $\Sigma^{W}$ cannot tolerate SCS outputs, then violates the safety requirements, i.e. if $\exists v_{i}, v_{i}^{f} \in V_{I}, \exists \psi \in \Psi, \Sigma\left(v_{i} / v_{i}^{f}\right)$, then $f\left(\Sigma \| \Sigma^{G}, v_{i}^{f}, v_{i}^{f} \rightarrow v_{o}, \psi\right)=$ true, but $f\left(\Sigma \| \Sigma^{W}, v_{i}, v_{i}^{f} \rightarrow v_{o}, \psi\right)=$ false.

For this problem, this paper presents a safety requirements-oriented interfaces environment (SRoIE) scheme: not only do SCS need to ensure the functional requirements, but also modules interfaces.

Definition 5. A SRoIE of a module is a tuple $\left\langle\psi, C I^{\psi}\right\rangle$, where: $\psi$ is a safety constraint; $C I^{\psi}$ is the core interfaces set of $\psi$, where $C I^{\psi}=\bigcup B_{i}, B_{i} \in K I^{\psi}$, and $B_{i}$ is the minimal set in $K I^{\psi}$, i.e. $\forall A_{j} \in K I^{\psi}$, if $B_{i} \bigcap A_{j} \neq \phi$, then $A_{j} \nsubseteq B_{i}$, $i, j \in N^{+}$.

The core interfaces set is monotone with respect to set inclusion, i.e. $\forall \Lambda_{1}, \Lambda_{2} \in$ $K I^{\psi}: \Lambda_{1} \subseteq \Lambda_{2} \Rightarrow\left(\Lambda_{1}\right.$ is core inter faces set $\Rightarrow \Lambda_{2}$ is core interfaces set $)$. SRoIE presents a minimal interfaces combination which could affect a specific safety constraint, and indicates implicitly a relation between interfaces and system safety. For example, if the key interfaces set of $\psi$ is $\left\{\left\{I_{1}, I_{2}, I_{3}\right\},\left\{I_{1}, I_{2}, I_{3}, I_{5}\right\}\right.$, $\left.\left\{I_{2}, I_{3}\right\},\left\{I_{4}, I_{5}, I_{6}\right\},\left\{I_{5}, I_{6}\right\}\right\}$, then the SRoIE is $\left(\psi,\left(\left(I_{2}, I_{3}\right),\left(I_{5}, I_{6}\right)\right)\right)$, which implies that if $\left\{I_{2}, I_{3}\right\}$ or $\left\{I_{5}, I_{6}\right\}$ fails, $\psi$ will be violated.

Through ensuring the SRoIE, safety in working environment can be ensured, and when a safety constraint is violated, maintenance information is provided that the states of interfaces corresponding to the constraint should be checked to prevent interfaces fault.

\subsection{SRoIE algorithm}

SRoIE can be deduced through model checking which is widely used in SCS to verify whether system satisfies a specific constraint or not $[7,8]$. In model checking framework, the gathering environment and working environment can be constructed in SCS functional model, then the fault tolerant ability of the two environments can be analyzed respectively by equations (5) and (6).

$$
R_{1}=f\left(\Sigma \| \Sigma^{G}, v_{i}^{f}, v_{i}^{f} \longrightarrow v_{o}, \psi\right)
$$




$$
R_{2}=f\left(\Sigma \| \Sigma^{W}, v_{i}^{f}, v_{i}^{f} \longrightarrow v_{o}, \psi\right)
$$

$\neg R_{1} \vee R_{2}=$ false means $\psi$ is satisfied in $E^{G}$, but violated in $E^{W}$. The counterexample $\mu_{n}$ returned by model checking can be divided into gathering environment case $\mu_{n}^{G}$ and working environment case $\mu_{n}^{W}, \mu_{n}^{G} \cup \mu_{n}^{W}=\mu_{n}$. Meanwhile, $\exists i, j \in N, 1 \leq i, j \leq$ num, $\forall\left(I_{i}, v_{i}\right) \in \mu_{n}^{W}, \exists_{1}\left(I_{j}, v_{j}\right) \in \mu_{n}^{G}, I_{i}=$ $I_{j}$, and $\forall k \in N, 1 \leq k \leq$ num, if $k \neq j$, then $I_{k} \neq I_{j}$.

$\exists i, j \in N, 1 \leq i, j \leq n u m,\left(I_{i}^{G}, v_{i}^{G}\right) \in \mu_{n}^{G},\left(I_{i}^{W}, v_{i}^{W}\right) \in \mu_{i}^{W}$, if $I_{i}^{G}=I_{i}^{W}$, $v_{i}^{G} \neq v_{i}^{W}$, then $\cup I_{i}^{G}$ is the key interfaces set of the safety constraint. SRoIE can be refined through the key interfaces set, and the SRoIE generation algorithm is shown in algorithm 1. Utilizing system functional model and safety constraint set, algorithm identifies the key interfaces set for every safety constraint by KIGeneration algorithm, then SRoIE is refined by CIRefinement algorithm.

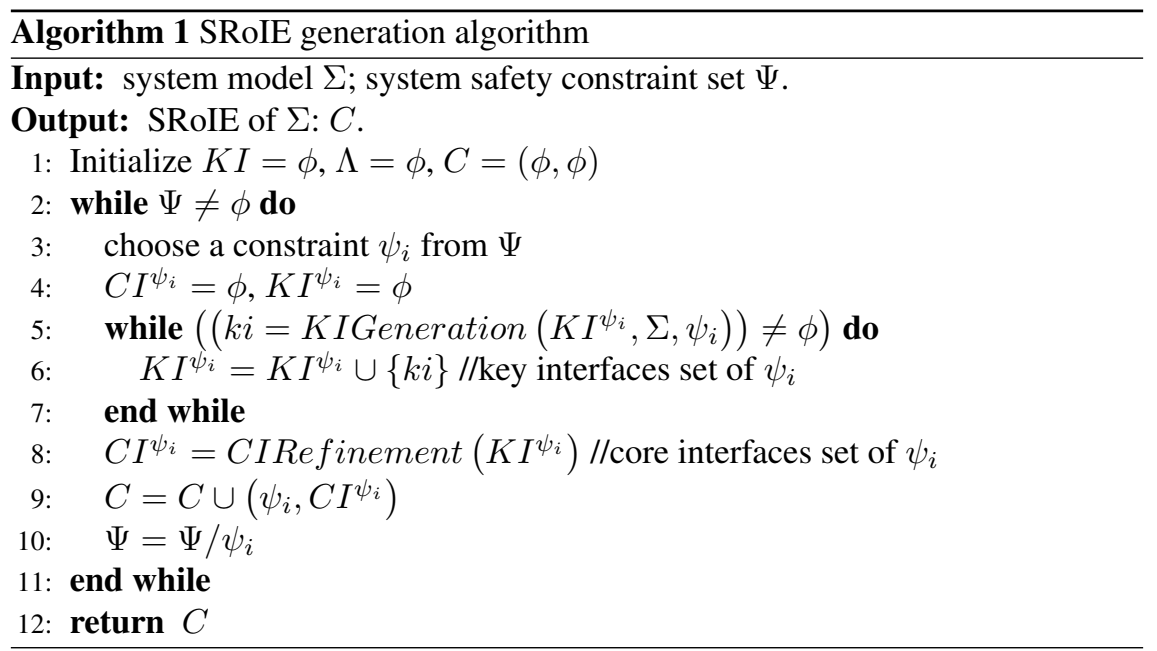

KIGeneration identifies the rest key interfaces based on key interfaces have been identified, which ensures the completeness of identification process for a specific constraint and is shown in algorithm 2 .

Since the key interfaces space is huge and redundant, a refinement for SRoIE is necessary, which is algorithm 3 CIRefinement. To reduce the computation efforts, the monotone characteristic of core interfaces set has been utilized.

\section{Case study}

Computer based interlocking (CBI) [9] is a key subsystem in railway signal domain. CBI undertakes a responsibility of arranging routes for trains movements in safety, and needs a strict fail-safe requirement $[10,11]$. As the effects of concurrency, conflict, and competition, EIF may easily cause a accident and affect 

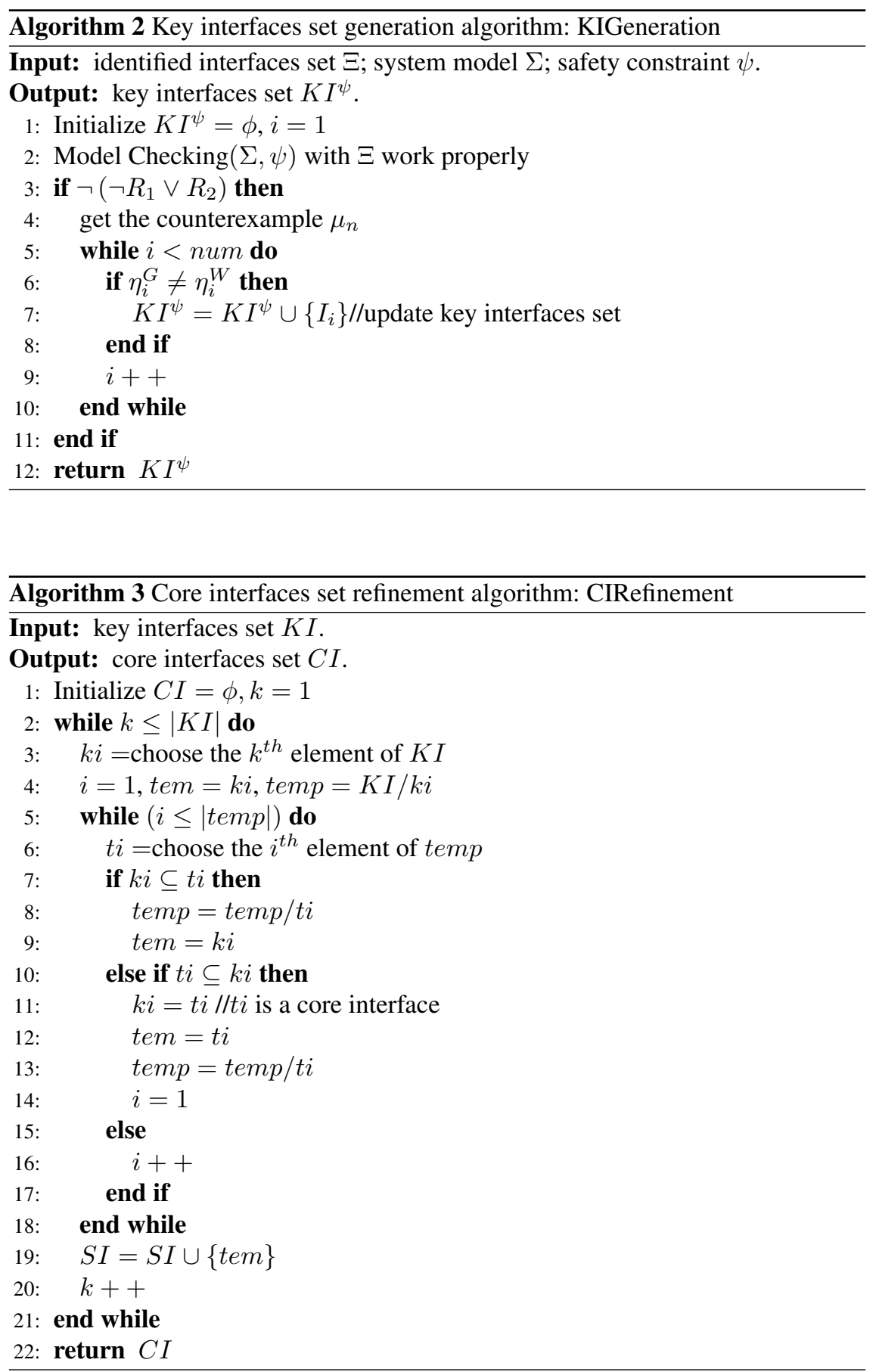
the safety and availability of signal system. The identification of SRoIE has a significance for CBI safety design and maintenance.

\subsection{Signal opening module}

Signal opening is a core functionality of CBI, and its safety directly affects the safety of movements of trains. For example, a line of a subway is shown in figure 1 . There is a point $P 04$ within the protected section of a downlink signal $X 0404$, so the straight route $X 0404 S R$ and diverging route $X 0404 D R$ can be set respectively depending on the point position.

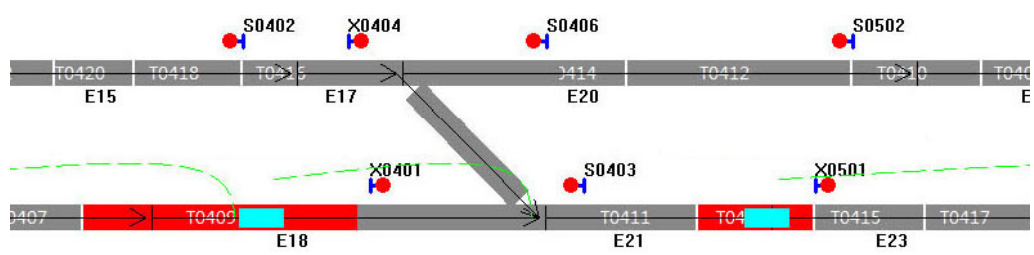

Figure 1: A subway line.

The signal opening module of X0404 $\Sigma_{X 0404}$ involves 27 input interfaces, including section, platform screen door, and point. $\Sigma_{X 0404}$ has four states: (SR_false, SR_true, DR_false, DR_true), and two mutually exclusive processes: $X 0404 S R$ and $X 0404 D R$. X0404SR has a initial state $S R_{-} f a l s e$, while $X 0404 D R$ has $D R_{-}$false. When corresponding sections are clear $(* P)$, platforms has no stop commands $(* S T O P)$, signal has no call-on command $(* C R)$, platform screen door is safe $(* S D)$, signal aspect is in safe state $(* S G Y A)$, depending on the position of P04 (P04SwNC for $X 0404 S R$, $P 04 S w R C$ for $X 0404 D R), \Sigma_{X 0404}$ transits to state $S R \_t r u e$ or DR_true.

\subsection{SRoIE identification and result analysis}

$\Sigma_{X 0404}$ needs to satisfy the constraint: the straight route and diverging route cannot be set simultaneously, denoted by: $\psi_{1}:=$ not $(X 0404 S R$ and $X 0404 D R)$. This paper utilized SCADE [12] to implement the SRoIE algorithm, and 109 key interfaces have been identified, for example ( $S T 0402 P, P 04 S w N C, P 04 S w R C)$, (P04SwNC,X0404SD,P04SwRC), (P04SwRC,X0404SGY A,P04SwNC, $042 S D)$. Then the SRoIE is refined: $\left(\psi_{1},(P 04 S w N C, P 04 S w R C)\right)$, which indicates:

1. $\left(\Sigma_{X 0404} \| \Sigma^{W}\right) \vDash \psi_{1}$, if the interfaces $P 04 S w N C$ and $P 04 S w R C$ have been assured;

2. When $\left(\Sigma_{X 0404} \| \Sigma^{W}\right) \not \models \psi_{1}$, the states of $P 04 S w N C$ and P04SwRC should be checked.

The safety interfaces identified through field experiences includes $S T 0402 P$, $T 0414 P, P 0414.0402 S w N C, 09 S D, P 04 S w R C$, etc, including the two core 
interfaces identified by SRoIE for $\psi_{1}$. Meanwhile, the rest of experience interfaces are identified at other safety constraints. The SRoIE result is consistent with the practical engineering experience, and the identification comprehensiveness is ensured. Moreover, the CBI interfaces have been associated with specific safety constraints respectively, and the faults modes that CBI should be ensured are suggested implicitly.

\section{Conclusion}

This paper divided the SCS environment into gathering environment and working environment. To ensure the safety in the two environment on system level, a SRoIE scheme is presented. Based on the system model and safety requirements, SRoIE scheme presents interfaces environment that a specific safety constraint should be ensured. The interfaces information can be used to improve the design or integration of modules, and guide system maintenance. SRoIE scheme has a guiding and practice significance for the design, development and maintenance of SCS.

\section{Acknowledgements}

This work is partially supported by the China Railway Corporation under Grant 2015X009-D and 2014X008-A. The authors also gratefully acknowledge the helpful comments and suggestions of the reviewers, which have improved the presentation.

\section{References}

[1] Knight J. C. Safety critical systems: challenges and directions. Software Engineering, 2002. ICSE 2002. Proceedings of the 24th International Conference on, pp. 547-550, 2002.

[2] U.S. NRC. Backgrounder on the Three Mile Island Accident. http://www.nrc. gov/reading-rm/doc-collections/fact-sheets/3mile-isle.html

[3] Elmqvist J. \& Nadjm-Tehrani S. Safety-oriented design of component assemblies using safety interfaces. Electronic Notes in Theoretical Computer Science, 182: pp. 57-72, 2007.

[4] Elmqvist J., Nadjm-Tehrani S. \& Minea M. Safety interfaces for componentbased systems. Computer Safety, Reliability, and Security. Springer Berlin Heidelberg, pp. 246-260, 2005.

[5] Elmqvist J. \& Nadjm-Tehrani S. Formal support for quantitative analysis of residual risks in safety-critical systems. High Assurance Systems Engineering Symposium, 2008. HASE 2008. 11th IEEE, pp. 154-164, 2008.

[6] Ying Liu \& Xu Zhongwei. Safety interface scheme for component-based safety critical software. Journal of Computer Applications, 28(11): pp. 2933 2935, 2008. 
[7] Clarke E. M., Grumberg O. \& Peled D. Model checking. MIT press, 1999.

[8] Baier C. \& Katoen J. P. Principles of model checking. Cambridge: MIT press, 2008.

[9] Hui M. A. Formal Modeling and Verification of Urban Rail CBTC Interlocking Based on CSP. Beijing Jiaotong University, 2013.

[10] Haifeng Wang, T. H. Xu et al. Novel Online Safety Observer for Railway Interlocking System. The Journal of Transportation Engineering ASCE, 139(7): pp. 719-727, 2013.

[11] Yang Yang \& Shaowen Zou. Design and Development of Computer-based Interlocking System SWJTU-II. Journal of the China Railway Society, 27(3): pp. 118-123, 2005.

[12] ESTEREL Technologies. SCADE Suite. http://www.esterel-technologies. com/products/scade-suite/ 